\title{
LFER and the Effect of Temperature on Oxyanion Adsorption by Goethite
}

\author{
Anna Dabizha ${ }^{1}$, Nataliya Vlasova $^{2}$, and Michael Kersten ${ }^{1, *}$ \\ ${ }^{1}$ Geosciences Institute, Johannes Gutenberg-University, 55099 Mainz, Germany \\ ${ }^{2}$ Institute of Surface Chemistry, National Academy of Sciences of Ukraine, $03164 \mathrm{Kiev}$, Ukraine
}

\begin{abstract}
A linear relationship between the Gibbs free energy, $\Delta G_{\mathrm{r}, \mathrm{H}+}$, of the aqueous complex deprotonation reaction, and the Gibbs free energy, $\Delta G_{\mathrm{r}, \mathrm{ads}}$, of bidentate surface complexation reaction of oxyanions was derived from modelling of temperature dependent batch equilibrium adsorption experiments. As exemplified in this study, this relationship may be exploited to predict temperature-dependent adsorption behavior of oxyanions not yet known such as pertechnetate.
\end{abstract}

\section{Introduction}

Despite a growing number of studies on surface complexation modeling, not much is known currently about the effect of temperature on surface complexation reactions. Attempts to derive thermodynamic constants from temperature dependent isotherm data often found in literature are inconsistent with thermodynamic principles. Thermodynamic data like Gibbs free energy should only be derived from chemical reactions rather than of simple distribution constants. Data for temperature-dependent oxyanion adsorption by goethite have already been reported from our laboratory [1-3]. The data suggest that the higher the temperature, the less amount of oxyanion is adsorbed on the Fe oxyhydroxide surface, i.e. that the adsorption reaction is exothermic. The decrease of surface acidity $\left(\mathrm{pH}_{\mathrm{pzc}}\right)$ with temperature results in a weaker screening of the negative charge of the surface complex, i.e., more repulsion occurs at higher temperature. This leads ultimately to a shift in the oxyanion adsorption envelope by about $0.2 \mathrm{pH}$ units per $10{ }^{\circ} \mathrm{C}$ towards the more acidic region, and thus in a weakening of the overall adsorption affinity with increasing temperature at circumneutral $\mathrm{pH}$ conditions. The data gained from temperature-dependent batch equilibrium adsorption experiments, if fitted by advanced surface complexation models like the CD-MUSIC, provide accurate Gibbs free energy values, $\Delta G_{\mathrm{r}}$,ads, of surface complexation reactions. This approach also allows for a linear free energy relationship (LFER) between the Gibbs free energy, $\Delta G_{\mathrm{r}, \mathrm{H}+}$, of the aqueous complex deprotonation, and

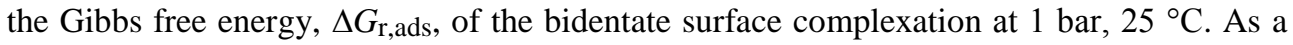
next step, this relationship may be exploited to predict the behavior of oxyanions not yet studied like pertechnetate as exemplified in this study.

\footnotetext{
*Corresponding author: kersten@uni-mainz.de
} 


\section{Materials and Methods}

\subsection{Model Approach}

A simple model approach often reported in literature is to determine the impact of temperature on adsorption isotherms by applying an equation for a "free energy of adsorption reaction", $\Delta G_{\mathrm{r}}{ }^{\circ}=-\mathrm{R} T \ln K_{\mathrm{D}}$, with the $K_{\mathrm{D}}$ value as the distribution coefficient or ratio between total adsorbed and total dissolved ion concentration. The latter ratio is commonly derived from the slopes of the Freundlich or Langmuir adsorption isotherm at different non-standard state temperatures. Apparent enthalpy values are then calculated from the slope of a van't Hoff plot $2.3 \log K_{\mathrm{D}}$ vs. 1/T. In principle, however, this approach cannot be extrapolated to any $\mathrm{pH}$ other than that used for derivation of the adsorption isotherms. Moreover, this simplyfied approach neglects that both the dissolved and adsorbed oxyanion speciation is commonly not unique but may comprise different species. The surface speciation may even change with temperature [3]. Therefore, such an approach is merely pseudo-thermodynamic and may lead to misleading predictions.

For derivation of adsorption enthalpies on an accurate thermodynamic basis, a chemical reaction approach such as the CD-MUSIC surface complexation model developed by Hiemstra \& Van Riemsdijk [4] has to be applied. Based on a bidentate surface complex structure commonly derived for oxyanions from spectroscopic EXAFS and ATR-FTIR measurements, a chemical adsorption reaction stoichiometry may be formulated as, e.g., for the case of selenate [3]:

$$
2 \equiv \mathrm{FeOH}^{-0.5}+\mathrm{SeO}_{4}^{2-}+2 \mathrm{H}^{+} \leftrightarrow(\equiv \mathrm{FeO}) \frac{\Delta z_{0}}{2} \mathrm{SeO}_{2}^{\Delta z_{1}}+2 \mathrm{H}_{2} \mathrm{O}
$$

For this bidentate surface complexation reaction, two moles of water per mole of oxyanion are released into solution, as suggested for a bidentate surface complexation [4]. The innersphere characteristics of this surface complex is represented by shifting half of its charge from the outer into the inner surface layer (or $0.10-0.25$ v.u. in case of neutral charged oxyanion species), neglecting any temperature dependence of the charge distribution.

Fitting the data previously gained for silicic acid, arsenite, and selenate in the temperature range $10-80{ }^{\circ} \mathrm{C}$ from batch equilibrium experiments (more than 300 experimental data points for each of the three oxyanions) has led to a unique set of surface complexation constants $\log K_{\mathrm{T}}$ [1-3]. The intrinsic $\log K_{\mathrm{T}}$ constants thus derived for different non-standard state temperatures were shown to change linearly with inverse temperature (1000/T when using the Kelvin scale). With the reasonable assumption of constant entropy $\Delta_{\mathrm{r}} S$ within the limited temperature range thus studied, this behavior of equilibrium constants can be represented by the van't Hoff two-term equation:

$$
R \cdot \log K_{T} \cdot \ln 10=\Delta_{r} S^{0}-\frac{\Delta_{r} H^{0}}{T}
$$

There is at least no problem to derive from the slope $2.3 \log K_{\mathrm{T}}$ vs. $1000 / \mathrm{T}$ a reliable standard enthalpy term, $\Delta H_{\mathrm{r}}{ }^{0}$. However, extrapolation to the y-axis intersect far from the few points thus gained is less reliable for derivation of an accurate entropy term. Nonetheless, the values for the entropy effect gained in this way for the three oxyanions yet studied were in a quite narrow range of $\Delta S_{\mathrm{r}}^{0}=94 \pm 20 \mathrm{~J} \mathrm{~mol}^{-1} \mathrm{~K}^{-1}$. This range appears reasonable if to consider an entropy value of $50 \mathrm{~J} \mathrm{~mol}^{-1} \mathrm{~K}^{-1}$ proposed from theoretical considerations for the loss of one water molecule upon the neutralization of one $-\mathrm{OH}$ surface site by a proton (or twice for a bidentate inner-sphere oxyanion surface complexation common for oxyanions) [5]. 


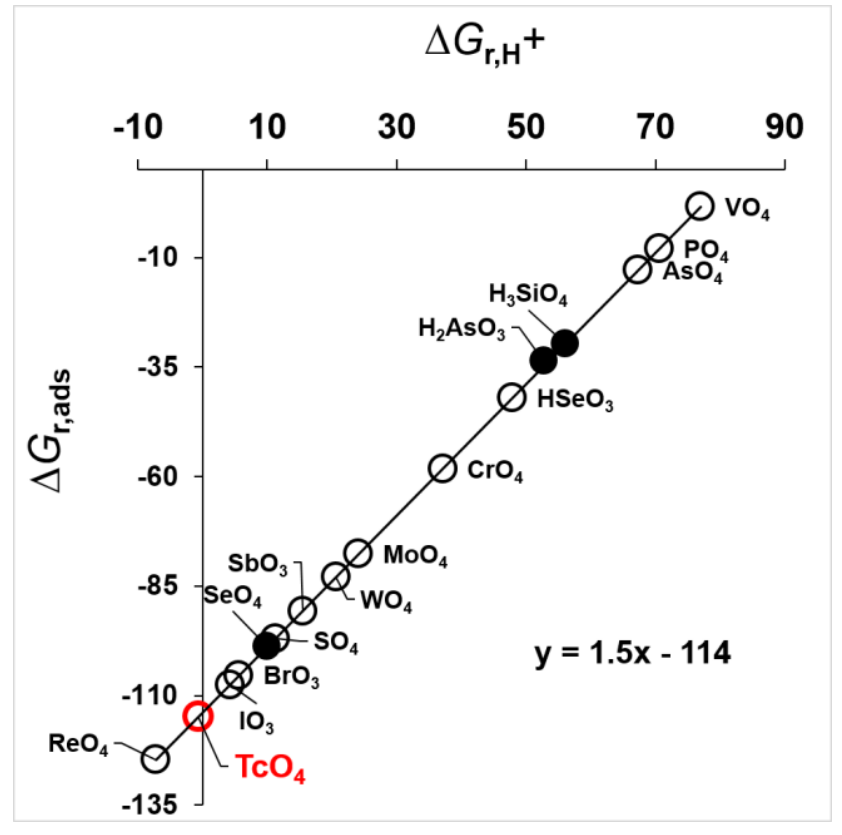

Fig. 1: Hypothetical linear relationship between the Gibbs free energy, $\Delta G_{\mathrm{r}, \mathrm{H}+}$, of the aqueous deprotonation reaction, and the Gibbs free energy, $\Delta G_{\mathrm{r} \text {,ads, }}$ of the bidentate adsorption reaction of oxyanions at $1 \mathrm{bar}, 25^{\circ} \mathrm{C}$. This LFER hypothesis is currently based on three black dots representing data gained so far on binary oxyanion/goethite systems [1-3]. The $\Delta G_{\mathrm{r}, \text { ads }}$ values of all open dots are predicted on basis of this correlation and known $\Delta G_{\mathrm{r}, \mathrm{H}+}$ values.

\section{Results and Discussion}

The results of the previous studies are compiled as black dots on the plot depicted in Fig. 1. This plot represents the LFER between the Gibbs free energies of reaction, $\Delta G_{\mathrm{r}, \text { ads }}$, for adsorption of the oxyanion of an element A,

$$
2 \equiv \mathrm{FeOH}+\mathrm{H}_{\mathrm{n}} \mathrm{AO}_{\mathrm{m}}{ }^{\mathrm{x}-}+(2-\mathrm{n}) \mathrm{H}^{+}=(\equiv \mathrm{FeO})_{2} \mathrm{AO}_{\mathrm{m}-2}+2 \mathrm{H}_{2} \mathrm{O}
$$

and Gibbs free energies of reaction, $\Delta G_{\mathrm{r}, \mathrm{H}+}$, for the aqueous deprotonation reaction of the respective oxyanionic acid,

$$
\mathrm{H}_{\mathrm{n}} \mathrm{AO}_{\mathrm{m}}=\mathrm{H}_{\mathrm{n}-1} \mathrm{AO}_{\mathrm{m}}{ }^{1-}+\mathrm{H}^{+}
$$

The constants for the latter reaction are compiled in thermodynamic handbooks. The three black dots for selenate, arsenite, and silicic acid provide the basis for a linear correlation between both Gibbs free energies (Fig. 1). Note, however, that accuracy of this LFER relationship is not yet sufficient, because two of the black data points are quite close to each other. More experiments to derive at missing critical points (e.g., for chromate and vanadate) are underway to validate this relationship at higher accuracy.

The LFER relationship could be used to infer at first approximation the standard molar Gibbs free energies of bidentate adsorption reaction for other oxyanions. For example, no experimental data on the temperature dependence of adsorption are yet available for the pertechnetate oxyanion, in spite of its importance for long-term prediction of the fate of the 
fission product Tc-99 in nuclear waste repositories. Although at circumneutral conditions a relatively weakly adsorbing oxyanion, bidentate surface complexation for pertechnetate has recently been evidenced by FTIR measurements in the acidic $\mathrm{pH}$ range (Foerstendorf, HZDR, pers. comm.). Based on this spectroscopic information, we suggest a CD-MUSIC surface complexation model for the bidentate adsorption reaction of pertechnetate according to the stoichiometry:

$$
2 \equiv \mathrm{FeOH}^{-0.5}+\mathrm{TcO}_{4}^{-}+2 \mathrm{H}^{+} \leftrightarrow(\equiv \mathrm{FeO})_{2}^{\Delta z_{0}} \mathrm{TcO}_{2}^{\Delta z_{1}}+2 \mathrm{H}_{2} \mathrm{O}
$$

with a charge distribution of $\Delta x_{0}=0.5$ and $\Delta x_{1}=-0.5$ fixed for this inner-sphere surface complexation. The LFER relationship predicts an adsorption constant of $\Delta G_{\mathrm{r}, \text { ads }}=-114.9 \mathrm{~kJ}$ $\mathrm{mol}^{-1}$, or a $\log K_{\mathrm{ads}}=20.0$ assuming a $\Delta S_{\mathrm{r}}^{0}=100 \mathrm{~J} \mathrm{~mol}^{-1} \mathrm{~K}^{-1}$. Note, however, that oxyanions are commonly bound by both inner- and outer-sphere surface complexation, and that the latter is increasingly dominating the overall adsorption reaction with increasingly negative $\Delta G_{\mathrm{r}, \text { ads }}$ values (i.e., from top to bottom of the LFER curve in Fig. 1). This suggests also an increasing exothermic effect on the inner-sphere portion and thus weakening of the surface binding by an increasing shift towards outer-sphere complexation. As a next step, the same relationship has to be constrained on the outer-sphere complexation reactions, before accurate predictions on the overall adsorption vs. temperature behavior can be made.

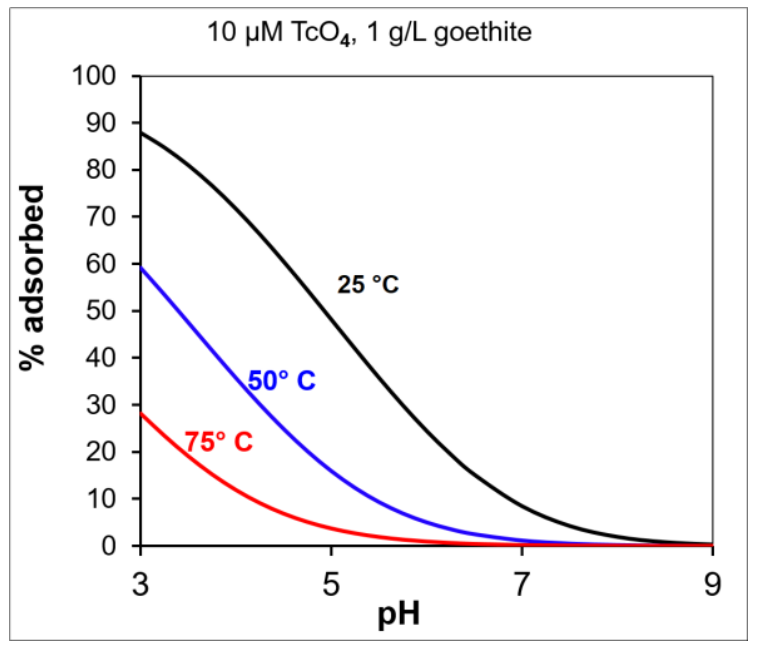

Fig. 2. Adsorption vs. pH curves at different temperatures predicted for the inner-sphere surface complexation part for pertechnetate adsorbed by goethite. The inner-sphere contribution to the overall adsorption mechanism is shifted significantly with increasing temperature towards the acidic $\mathrm{pH}$ range indicating a weakening of the overall adsorption reaction.

\section{References}

1. M. Kersten, N. Vlasova, Chem. Geol. 262, 372 (2009)

2. M. Kersten, N. Vlasova, Appl. Geochem. 24, 32 (2009)

3. M. Kersten, N. Vlasova, Radiochim. Acta 101, 413 (2013)

4. T. Hiemstra, W.H. van Riemsdijk, J. Colloid Interface Sci. 301, 1 (2006)

5. D.A. Kulik, Interf. Sci. Technol. 11, 171 (2006) 